\title{
Herausforderung Methodeneinsatz - Erklärungsansätze aus der Akzeptanzforschung
}

\author{
Simon J. Nicklas ${ }^{1 *}$, Anne Wallisch ${ }^{1}$, Kristin Paetzold ${ }^{2}$ \\ ${ }^{1}$ Universität der Bundeswehr München, Institut für Technische Produktentwicklung, München \\ 2 Technische Universität Dresden, Institut für Maschinenelemente und Maschinenkonstruktion, Dresden \\ ${ }^{*}$ Korrespondierender Autor: \\ Simon J. Nicklas \\ Universität der Bundeswehr München \\ Fakultät für Luft- und Raumfahrttechnik \\ Institut für Technische Produktentwicklung, LRT3 \\ Werner-Heisenberg-Weg 39 \\ 85579 Neubiberg \\ Telefon: +4989 6004-2391 \\ Mail:simon.nicklas@unibw.de
}

\begin{abstract}
Whilst there is broad agreement that methods can efficiently support development work, new methods often find little acceptance in industrial practice in terms of consistent use. Therefore, this paper aims to identify factors influencing the formation of acceptance that help successfully design and implement methods. To this end, a literature review of acceptance research identifies relevant models for product development and describes their application context, dominant and moderating variables, and explananda. Subsequently, a disciplinary-historical research map for the explanatory power of each acceptance-forming step is derived. Finally, this article deduces possible points of connection and requirements for the specific adaptation of the findings to methods as an object of acceptance.
\end{abstract}

\section{Keywords}

acceptance research, method application, method acceptance, theory concept 


\section{Motivation}

Das Hauptaugenmerk der Forschung in der Produktentwicklung liegt in der Unterstützung von dort typischen Aufgaben. Durch die wissenschaftliche, methodische Aufbereitung von Erkenntnissen zur Problemlösung wird eine effiziente Entwicklung unterstützt. Parallel dazu ist die Methodenentwicklung auch Teil der Arbeit in den Unternehmen. Der Fokus hier liegt darauf, das Erfahrungswissen der Mitarbeiter in der Breite zugänglich zu machen. Über die Implementierung von Methoden und Prozessen werden Best-Practices generalisiert und implizites Wissen wird explizit gemacht. Trotzdem ist gerade die Implementierung neuer Methoden für viele Unternehmen eine Herausforderung und stößt dort auf Widerstand [1]. Obwohl zwar einerseits anerkannt wird, dass Methoden die Entwicklungsarbeit effizient unterstützen können, zeigen Studien gleichzeitig, dass insbesondere komplexe Methoden gerade in der industriellen Praxis häufig keine Akzeptanz finden [2]. Als Akzeptanz wird nach HILBIG [3] in diesem Kontext eine mehr oder weniger zustimmende Einstellung eines Individuums gegenüber einem Artefakt oder einem anderen Sachverhalt verstanden.

Um die Akzeptanz speziell für die Methodeneinführung bewerten und anschließend den Einführungsprozess selbst aktiv unterstützen zu können, erscheint es zunächst zielführend, bereits bekannte, allgemeinere Ansätze aus der Akzeptanzforschung zu nutzen und an den spezifischen Kontext der Methodenanwendung in der Produktentwicklung anzupassen. Hieraus ergibt sich die Forschungsfrage:

\section{Welche Modelle aus der Akzeptanzforschung helfen uns zu erklären, wie neue Methoden und Tools in die Anwendung kommen können?}

\section{Verwendete Methoden und Vorgehensweise}

Die Beantwortung der Forschungsfrage erfordert eine umfassende Literaturstudie zu existierenden Akzeptanzmodellen aus verschiedenen Fachrichtungen, deren Aufbau und Vorgehensweise im Folgenden skizziert wird und in deren Verlauf entwicklungsrelevante Ansätze identifiziert wurden, die sowohl Akzeptanzmodelle und deren zum Teil problemspezifische Weiterentwicklungen betrachten (z.B. [4], [5]) aber auch einzelne spezifische Aspekte vertiefend diskutieren (z.B. [6]). Zusätzlich werden Prozessmodelle zur Akzeptanzabsicherung näher untersucht [7]. Darauf folgt die Beschreibung der in der Analyse zu berücksichtigenden Modelle anhand ihrer jeweiligen Akzeptanzkontexte, erklärten Strukturvariablen und ihres Prozesscharakters als Stand der Technik in Abschnitt 3. Davon ausgehend werden Überlegungen angestellt, wie diese Einsichten hinsichtlich ihrer Eignung zur Erklärung für die Bildung von Methodenakzeptanz im Kontext der Produktentwicklung zu bewerten sind (Abschnitt 4), was wiederum Rückschlüsse auf die notwendige, spezifische Anpassung erlaubt. Auf Basis typischer Denkabläufe des Entwicklers zur Problemlösung werden schließlich die Erkenntnisse hinsichtlich der Akzeptanzsteigerung in der Methodenentwicklung und -einführung verdichtet und diskutiert (Abschnitt 5). In einem letzten Abschnitt (6) folgt der Ausblick auf weiterführende Untersuchungen, um den Methodeneinführungsprozess künftig strukturiert zu unterstützen.

\subsection{Untersuchungsplanung}

Der Zugang zu Gegenstand und Feld der Akzeptanzforschung erfolgt über eine Literaturrecherche mittels strukturierter Datenbankanalyse. Die Treffer der Stichwortanalyse sollten in einem vierstufigen Verfahren (Analyse der Abstracts, Wiederholung der Stichwortsuche im Fließtext, Kontextanalyse der Fundstellen, Inhaltsanalyse des Dokuments) in die Feinanalyse aufgenommen werden. Je nach Trefferquote sollten die Suchkriterien im weiteren Forschungsverlauf verfeinert und jede Anpassung des Suchschemas entsprechend dokumentiert werden. Als Datenquelle standen die wissenschaftlichen Datenbanken Scopus sowie Mendeley zur Auswahl. 


\subsection{Datensammlung}

Die ungefilterte Stichwortsuche nach „Akzeptanz“ brachte 1832 Ergebnistreffer in Mendeley, die Erweiterung „Akzeptanz+Produktentwicklung“ reduzierte die Trefferanzahl auf 17. Der Suchbegriff „Methodenakzeptanz“ führt zu zwei Treffern. Scopus lieferte für den Begriff „Akzeptanz“ eine Trefferzahl von 766 Dokumenten, für „Akzeptanz+ Produktentwicklung“ sowie "Methodenakzeptanz" keine Treffer. Aufgrund der besseren Filterwerkzeuge wurde die Recherche via Scopus fortgesetzt. Das Durchsuchen der Trefferartikel nach dem Kontextbegriff „Technik“ lieferte 36 Dokumente. Die Durchsicht gab Hinweise auf Literatur aus dem englischsprachigen Raum. Entsprechend wurden die bisherigen Suchbegriffe übersetzt und aufgrund des zu erwartenden großen Datenvolumens direkt in Verknüpfung gesucht. Die 464 resultierenden Ergebnisse wurden in einem nächsten Schritt auf die Disziplinen Engineering, Computer-, Social Sciences sowie Business, Management and Accounting sowie die Stichworte Product Development und Product Design gefiltert. Die Analyse der verbleibenden 60 Treffer reduzierte sich nach Durchsicht auf Eignung um 39, deren Inhalte und Quellenverzeichnisse als Datenbasis für die Recherche nach Akzeptanz erklärenden Modellen dienten. Um entsprechende Arbeiten auch für den deutschsprachigen Raum identifizieren zu können, wurden alle entsprechenden Suchbegriffe noch einmal via Google Scholar und Springer Link gesucht. Auch die Quellenverzeichnisse dieser Trefferliste (31) wurden für die gezielte Recherche nach Erklärungsmodellen für Akzeptanz im Technikumfeld genutzt. Um empirische Studien ohne theoretische Weiterentwicklung bereinigt umfasst die finale Datenbasis 33 Akzeptanzmodelle. Diese wurden in der folgenden Feinanalyse auf die innen zugrundeliegende Definition von Akzeptanz, Kontext, Akzeptanzsub- und -objekte, ihre Herkunftsdisziplin sowie Zielstellung untersucht.

\subsection{Datenanalyse}

Die Ergebnisse der Feinanalyse wurden zunächst deskriptiv ausgewertet. Diese Auswertung umfasst zentrale Gemeinsamkeiten und Unterschiede im methodischen Ansatz der Modelle, in ihren Frage- und Zielstellungen, Betrachtungsausschnitten, beschriebenen Einflussgrößen, Erklärungslücken und ihrer Aussagekraft. Eine der einzuhaltenden Formatvorgabe entsprechend knappe Zusammenfassung der identifizierten Modelle findet sich in Abschnitt 3. Aus diesen deskriptiven Ergebnissen wurden relevante Funde mithilfe des theoretischen Unterbaus von ROGERS [7] verdichtet und schließlich mit den Spezifika des Methodeneinsatzes in der Produktentwicklung synthetisiert. Die Ergebnisse werden in den Abschnitten 4 und 5 aufbereitet. Abschluss findet diese Analyse mit einer kritischen Diskussion und Reflektion ihrer Limitierungen und dem Aufzeigen weiterer Forschungsbedarfe.

\section{Akzeptanzmodelle und -kontexte}

Technologieakzeptanzforschung umfasst Fragestellungen, die sich mit unterschiedlichen Aspekten wie der individuellen Nutzerakzeptanz größerer und kleinerer technischer Artefakte (bspw. Assistenzroboter und Chatbots), bis hin zur gesellschaftlichen Akzeptanz neuer bzw. risikobehafteter Technologien (etwa im Kontext der Digitalisierung und Künstlicher Intelligenz) befassen. Die entsprechenden Modelle werden in der Literatur regelmäßig nach ihrem Aufbau unterschieden (u.a. [8-10]), wobei im Folgenden auf die vier Arten eingegangen wird, wie sie auch bei DILLER [11] aufgeführt werden:

- Input-Modelle (I) beschreiben ausschließlich die vorgeschalteten Einflussfaktoren auf die Akzeptanz.

- Input/Output-Modelle (I/O) erfassen zusätzlich die dem Akzeptanzprozess nachgelagerten Auswirkungen auf das Verhalten des Nutzers. 
- Rückkopplungsmodelle $(\boldsymbol{R})$ schließen den Kreis und beschreiben vornehmlich die Auswirkungen des Akzeptanz- und Anwendungsprozesses auf die ursprünglich zugrunde gelegten Einflussfaktoren.

- Aus so genannten dynamischen Akzeptanzmodelle (D) lassen sich zum einen bereits Empfehlungen für die Gestaltung ableiten, zum anderen sind diese bemüht, unterschiedliche Entwicklungen im individuellen Akzeptanz- und Nutzungsprozess abzubilden, um so den dichotomen Akzeptanzbegriff aufzulösen.

Die von SCHÄFER \& KEPPLER [12] alternativ vorgeschlagene Kategorie der Phasenmodelle, die an der Aufteilung des Akzeptanzprozesses in mehrere Phasen und gegebenenfalls weitere Unterabschnitte zu erkennen sind, scheint für die vorliegende Analyse weder notwendig noch trennscharf genug, so dass diese nicht weiter betrachtet wird. Vielmehr erscheint es sinnvoll, zwischen der Struktur- und der Prozessbetrachtung zu unterscheiden. Auf der einen Seite wird die Struktur durch die verschiedenen Einflussfaktoren des jeweiligen Modells und der enthaltenen kausalen Abhängigkeiten bestimmt, auf der anderen Seite folgt jeder Ansatz einem zeitlichen Zusammenhang, der den zugrundeliegenden Prozess beschreibt. In der Regel werden beide Sichtweisen in einem logischen Gesamtmodell zusammengeführt, das jedoch je nach Zielstellung unterschiedliche Schwerpunkte setzen kann. Die jeweilige Zuordnung der folgenden Modelle zu oben genannten Modellarten ist der Übersicht halber in Tabelle 1 zusammengefasst.

Ihren Ursprung findet die Akzeptanzbetrachtung auf verschiedenen Teilgebieten der Psychologie und Soziologie. So entstand aus einer Betrachtung der Adoption oder NichtAdoption von Innovationen im Agrarsektor die Innovationsdiffusionstheorie (IDT) nach ROGERS [13], die insbesondere im Kontext sozialwissenschaftlicher Fragestellungen intensiv bearbeitet, angewendet und verfeinert wurde und in ihrer aktuellen Form [7] sowohl den zeitlich logischen, individuell-persönlichen Adoptionsprozess, die Verbreitung von Innovationen auf gesellschaftlicher Ebene, als auch die Integration innovativer Technologien und Anwendungen auf Organisations- und Unternehmensebene beschreibt. Zudem liefert das Modell mit den explizit aufgeführten, alternativen Ergebnissen des Akzeptanzprozesses, von der Adoption hin bis zur Ablehnung, einen ersten Ausblick auf spätere dynamische Akzeptanzmodelle. Als spezifischer Ansatz findet sich zu einer ähnlichen Zeit in der Psychologie auch die PARTheory, welche sich zunächst auf die Akzeptanz und Ablehnung in einer Eltern-Kind-Beziehung beschränkte [14] und ab dem Jahr 1999 zu einer allgemeinen Vorhersage und Erklärung von Konsequenzen im Bereich zwischenmenschlicher Ablehnung und Zustimmung ausgebaut wurde [15] und später im KI-Akzeptanzmodell (s.u.) wieder aufgegriffen wurde. Die Verknüpfung zwischen persönlicher Einstellungen, subjektiver Normen und daraus resultierendem, bewusstem Verhalten stellt die Theory of Reasoned Action (TRA) [4] her und wurde von AJZEN [16] unter dem Namen Theory of Planned Behavior (TPB) vor allem hinsichtlich der wahrgenommen Verhaltenskontrolle als Einflussgröße auch auf unbewusstes Verhalten erweitert und kurz darauf nochmals überarbeitet [17]. Parallel dazu entstand das umfangreiche Model of PC Utilization (MPCU) nach TRIANDIS [18], das zunächst die Effekte einer Reihe von sozialen und strukturellen Kontextfaktoren auf die Nutzung von PCAnwendungen in Organisationen beschreibt und ähnlich wie auch TRA und TBP später von weiteren Autoren, insbesondere aus dem angloamerikanischen Raum, aufgegriffen und angepasst wurde. In Anbetracht der vielfältigen Modelle mit spezifischen Stärken und Schwächen ist der Versuch, verschiedene Ansätze wieder zusammenzuführen, nachvollziehbar: So veröffentlichten DAvIS u. a. [19] das Motivational Model (MM) als kombiniertes Modell aus TRA und TAM (s.u.), um die Nutzung von Computern am Arbeitsplatz besser erklären und Empfehlungen für die Softwaregestaltung ableiten zu können. Aus einer vergleichbaren Motivation heraus entstand auch die Beschreibung der Influence of Attitudes on Behavior [20] als Verknüpfung von TRA und TBP. Die Dual Process Theory of Cognition [6] liefert wiederum Ansätze, den persönlichen Entscheidungsprozess besser zu verstehen 
und erklärt diesen als Ergebnis zweier verschiedener innerer Systeme: eines autonom emotionalen und eines reaktiv rationalen.

Mit der zunehmenden Verbreitung und Verfügbarkeit von Arbeitsplatz- und Heimcomputern in den 80er und 90er Jahren des letzten Jahrhunderts entstanden aus der psychologischen Akzeptanzbetrachtung heraus noch spezifischere Modelle im Bereich der Human-ComputerInteraction und Wirtschaftsinformatik zur Beschreibung von Akzeptanz gegenüber Informationsmanagementsystemen. Bereits 1979 setzte sich ROBEY [21] in diesem Sinne mit CRM-Systemen auseinander und berücksichtigte im so genannten Model of User Behavior auch systemische Unternehmensfaktoren zur Beschreibung der Arbeitsleistung und deren Rückkopplung via extrinsischer und intrinsischer Belohnung auf die Eingangsfaktoren des Modells. Ausgehend von TRA entwickelte DAVIS [22] aus einer ähnlichen Motivation heraus das Technology Acceptance Model (TAM), welches mehrfach überarbeitet wurde, um sowohl die Einflussfaktoren für die wahrgenommene Nützlichkeit [5] in TAM2 als auch die wahrgenommene Benutzerfreundlichkeit [23] eines Systems in TAM3 detaillierter zu beschreiben. DEGENHARDT [24] berücksichtigt in seinem Modell wiederum vornehmlich individuell-persönliche Komponenten und führt zum ersten Mal explizit die „Aufgabenkompatibilität“ eines Kommunikationssystems ins Feld. THOMPSON u. a. [25] wählen einen abweichenden Ausgangpunkt: Sie bauen auf Triandis' Modell (s.o.) auf, vereinfachen die Eingangsfaktoren, überspringen die fehlerbehaftete Messung der Akzeptanz und legen den Fokus der Betrachtung stattdessen auf die Nutzungsphase. Durch die im Sinne einer einfacheren Validierung verkürzte Betrachtung wird das ursprüngliche Modell faktisch auf ein Input-Modell reduziert. Zusätzlich zu oben genannten Akzeptanzmodellen nach Davis, Bagozzi, Robey und Thompson beziehen sich COMPEAU \& HIGGINS [26], [27] explizit auf die sozialkognitive Lerntheorie (SCT) nach Albert Bandura und entwickeln daraus das Modell der Computer-bezogenen Selbstwirksamkeitserwartung (Computer Self-Efficacy) und beschreiben dessen Auswirkung auf die individuelle Arbeitsleistung. Kein Akzeptanzmodell im engeren Sinn, aber dennoch relevant im Sinne der Fragestellung des Beitrags, ist die Technology-to-Performance-Chain (TPC) von GOODHUE \& THOMPSON [28] als zweiteilige Betrachtung der Auswirkungen auf die Arbeitsleistung durch persönliche Einstellungen einerseits und den Task-Technology Fit (TTF) andererseits. Letzterer bezieht sich, ähnlich wie schon die Aufgabenkompatibilität bei Degenhardt, auf die Eignung einer Technologie für die angedachte Aufgabe und berücksichtigt dabei aufgabenbezogene, technologische und individuelle Charakteristika. Der Bezug zwischen Technologie und Aufgabe spielt auch eine zentrale Rolle im integrierten Akzeptanzmodell aus TAM und TTF von DISHAW \& STRONG [29], das die Varianz in der Nutzung von IT-Tools zu erklären versucht. Im deutschen Sprachraum wurde zudem das DART-Modell [30] entwickelt, das eine gezielte Erweiterung des ursprünglichen TAM um individuelle Kontextfaktoren darstellt und in dessen Rahmen ein zweites Modell präsentiert wird, das die Sicht desjenigen zeigt, der die Innovation einführt. Zeitgleich veröffentlichten VENKATESH u. a. [31] das UTAUT-Modell (Unified Theory of Acceptance and Use of Technology), um die vorangegangenen Entwicklungen auf dem Gebiet der Akzeptanzforschung wieder zusammenzuführen. In ihrem umfassenden Modell sind Erkenntnisse aus der Entwicklung und Anwendung von TRA, TAM, TAM2, MM, TPB, MPCU, IDT, SCT und eines kombinierten TAM-TPB-Ansatzes nach Taylor und Todd (1995) (ebd.) eingeflossen und sie beschreiben so einen umfangreichen Katalog an Einflussgrößen über vier zentrale Konstrukte (sozial, strukturell, leistungsorientiert, aufwandsorientiert) auf die Verhaltensintension und das tatsächliche Verhalten. Das ursprünglich enthaltende Konzept der Freiwilligkeit wurde in UTAUT2 fallen gelassen und stattdessen, neben anderen Anpassungen, die intrinsische, hedonistische Motivation eingeführt [32].

Die anfängliche Fixation auf IT-spezifische Fragestellungen innerhalb der Akzeptanzforschung hat sich spätestens seit der Jahrtausendwende gelöst und findet vermehrt Verbreitung in allgemeinen betriebswissenschaftlichen Betrachtungen. So erweitert KOLLMANN [9] das Betrachtungsfeld mit dem dynamischen Akzeptanzmodell auf 
Telekommunikations- und Multimediasysteme im Speziellen und Nutzungsgüter im Allgemeinen und versucht dabei, den dichotomen Akzeptanzbegriff aufzulösen und unterschiedliche (freiwillige) Nutzungsintensitäten zu erfassen. Die Kehrseite der Akzeptanzbetrachtung, nämlich den Widerstand gegen eine Innovation, ergänzen BAGOzZI \& LEE [33] aus Marketing-Sicht im Model for Consumer Resistance to, and Acceptance of Innovations in zwei separaten Prozessen bezüglich der persönlichen Zielsetzung und anschließenden Zielverfolgung. SWEENEY \& SOUTAR [34] greifen unter dem Titel Consumer Perceived Value das Konzept des vom Kunden wahrgenommenen Wertes eines Produktes auf und erörtern diesen auf allgemeiner Markenebene. Die Wertschätzung eines Kunden für ein Produkt messen sie auf einer 19-teiligen Erhebungsskala namens PERVAL. Die freiwillige Technologienutzung im heimischen Umfeld und im Besonderen die Verbreitung nach der anfänglichen Adoption beleuchten SHIH \& VENKATESH [35] im Use-Diffusion Model. Das dynamische Akzeptanz- und Wirkungsmodell [10] greift am Beispiel von Mobile-BusinessAnwendungen sowohl zeitliche Vorgänge innerhalb der Akzeptanzbildung als auch statische Einflussfaktoren samt zugehöriger Rückkopplungen auf. Vollständig auf den negativen Fall, nämlich dass eine Innovation abgelehnt wird, konzentriert sich die Consumer Resistance Hierarchy nach KLEIJNEN u. a. [36]: Dieses konzeptionelle Rahmenwerk ergründet die Hauptursachen des Widerstandes auf Verbraucherseite und liefert hierfür einen dreistufigen Erklärungsansatz von Prokrastination über Ablehnung bis hin zu aktivem Widerstand.

Parallel zur disziplinübergreifenden Verbreitung der Akzeptanzbetrachtung lässt sich auch die Entwicklung stark kontextspezifischer Modelle beobachten: So geht SCHLAG [37] der Fragestellung nach, wie eine nachhaltige Veränderung des Mobilitätsverhaltens mit Hilfe von Straßengebühren unterstützt werden kann und erarbeitet hierzu ein detailliert zugeschnittenes Rückkopplungsmodell. Spätestens wenn Technologien und Hilfsmitteln eine eigene Persönlichkeit zugeschrieben werden kann (siehe KI-Akzeptanzmodell), gewinnen auch die Untersuchungen von DEWALL \& BUSHMAN [38] für das vorliegende Thema an Relevanz. Diese stellen zwar kein Akzeptanzmodell im engeren Sinn dar, behandeln jedoch ausführlich die möglichen Folgen erfahrener sozialer Ablehnung aus medizinischer Sicht. Ebenfalls aus dem Bereich der Medizin stammt das Akzeptanzmodell für die Medizintechnik und Telemedizin nach BRUKAMP [39]. Mit der Zielstellung, ethische Problemfelder beim Einsatz neuer Technologien klären zu können, baut BRUKAMP auf einem vereinfachten UTAUT-Modell auf und konkretisiert dieses auf theoretischer Ebene mit Hilfe fachspezifischer Rahmenbedingungen und Einflussfaktoren. Das Akzeptanzmodell für Fahrassistenzsysteme (FAS) von ARNDT [40] basiert auf TPB und orientiert sich bezüglich des Aufbaus stark an SCHLAGs Akzeptanzmodell von Road-Pricing-Maßnahmen. Neben der obligatorischen Anpassung der Einflussfaktoren nimmt ARNDT zudem eine Kategorisierung der Variablen in Personenebene, Objektebene, Entscheidungsebene und Akzeptanzebene vor (S. 54-55). Das neuste und letzte Modell in dieser Aufstellung, das KI-Akzeptanzmodell (KIAM) [41], greift auf Input-Seite unter anderem die Ansätze zur Akzeptanz in zwischenmenschlichen Beziehungen nach ROHNER auf, um auch Effekte berücksichtigen zu können, die sich insbesondere für fortschrittliche Technologien basierend auf künstlicher Intelligenz ergeben, die zumindest teilweise als eigenständige Persönlichkeit wahrgenommen werden können. Erweitert wird dieses Konstrukt auf Input-Seite durch TAM3, sowie KI-spezifische Erweiterungen. Die logische Verknüpfung dieser Elemente erfolgt über die beiden Systeme der Dual Process Theory of Cognition. Im Gegensatz zu TAM3 erfolgt jedoch keine explizite Betrachtung der Systemnutzung.

\section{Einflussgrößen auf die Akzeptanzbildung}

In der Zusammenschau der Akzeptanzmodelle wird deutlich, dass bei der Betrachtung technischer Unterstützung in verschiedenen Situationen auch unterschiedliche Schwerpunkte gesetzt wurden. So verwundert es nicht, dass der Akzeptanzbegriff von den aufgeführten 
Autoren durchaus abweichend verwendet wird. Gerade in Modellen, die einen freiwilligen Nutzungskontext beschreiben, wird die Akzeptanz eines Artefakts häufig implizit oder explizit mit dessen Nutzung oder der vorausgehenden Nutzungsintention gleichgesetzt (z.B. bei [21], [25], [31], [32]), während andere Ansätze die Einstellung des Nutzers vom beobachtbaren Verhalten unterscheiden (siehe z.B. [4], [5], [16], [17], [22], [23]).

Zudem hat sich gezeigt, dass die Autoren zum Teil zu recht unterschiedlichen Einflussparametern kommen, die einerseits vom Akzeptanzobjekt abhängen und andererseits aus dem spezifischen fachlichen Blickwinkel heraus resultieren. Während die ersten Überlegungen zu Akzeptanzmodellen überwiegend von Psychologen und Sozialwissenschaftlern getrieben und weiterentwickelt wurden, erfolgte über die Zeit eine Diffusion in andere Disziplinen. Hier wurden diese mit dem spezifischen Kontext für spezifische Akzeptanzobjekte (Informationssysteme, medizinische Systeme etc.) adaptiert und weiterentwickelt. Jedes der betrachteten Akzeptanzmodelle scheint für die Untersuchung von Methodenakzeptanz durchaus interessante Aspekte beizusteuern. Um diese Einflussfaktoren zielorientiert zu strukturieren und bezüglich des Nutzens für die Beschreibung von Methodenakzeptanz bewerten zu können, wird auf die Diffusionstheorie nach RoGERS [7], [13] zurückgegriffen, der in einem Prozessmodell die Einführung und Verstetigung neuer Technologien über fünf Schritte beschreibt .

Dieser Diffusionsprozess gliedert sich dabei im Wesentlichen in zwei übergeordnete Phasen: die Initiierungsphase besteht aus den Schritten Kenntnisnahme (I.) und Überzeugung (II.). Die Mitglieder eines sozialen Systems erfahren dabei zum einen Probleme in der Durchführung von Aktivitäten und werden zum anderen hierfür mit neuartigen Lösungsansätzen konfrontiert. Im Ergebnis dieser Auseinandersetzung steht eine Entscheidung (III.) für Art und Umfang der Anwendung neuer Lösungsansätze, die es in der zweiten Phase, der Implementierungsphase einzuführen gilt. Positive Erfahrungen mit den Lösungsansätzen bei deren Implementierung (IV.) in den Alltag führen idealerweise im weiteren Verlauf zur Bestätigung (V.) der ursprünglich getroffenen Entscheidung. Bleibt diese aus, ist auch eine Anpassung der Entscheidung möglich.

Dieses Modell soll an hier als Referenz für die weiteren Betrachtungen verwendet werden - zum einen, da es sich hierbei um das älteste der nachstehend aufgeführten Akzeptanzmodelle handelt und es als breit angelegtes Modell sowohl soziale als auch individuelle Aspekte der Akzeptanzbildung berücksichtigt. Zum anderen wurde es in der Zeit seit seiner ersten Vorstellung laufend aktualisiert und in der Praxis validiert. Nichtsdestotrotz zeigt die folgende Analyse, dass dieses Modell um einen weiteren, vorgeschalteten Schritt ergänzt werden muss, der auch den Ursprung der vorherrschenden Rahmenbedingungen und Prädiktoren (0.) zu Beginn des eigentlichen Akzeptanzprozesses erfasst.

Der Vergleich der Akzeptanzmodelle in Tabelle 1 lässt erkennen, dass sich mit deren Hilfe jeweils unterschiedliche Schritte innerhalb des erweiterten Diffusionsprozesses erklären lassen. Nur wenige, hauptsächlich anwendungsbezogene Modelle decken alle Schritte (zumindest teilweise) ab. Insbesondere bei den frühen Modellen ist, wenig überraschend, eine "Tendenz zur Mitte“ des Prozesses zu erkennen - also der für die Akzeptanz zentralen Überzeugung und Entscheidung. Einen entgegengesetzten Ansatz verfolgt hier die Technology-to-Performance Chain, die sich besonders mit den Rahmenbedingungen des Prozesses und den resultierenden Folgen aus dem Adoptionsschritt auseinandersetzt.

Eine Sonderstellung nimmt die Interpersonal Acceptance-Rejection Theory in ihren zwei Ausprägungen [14], [15] sowie die Theorie der Social Acceptance and Rejection [38] ein. Aufgrund der jeweiligen Forschungsschwerpunkte weichen diese für sich allein genommen im strukturellen Aufbau zu weit ab, um eine Zuordnung der Schritte in Tabelle 1 sinnvoll zu ermöglichen. Die Zusammenhänge für die technologische oder methodische Akzeptanz ergeben sich erst durch umfangreiche Anpassungen und die Einbettung in einen entsprechenden Rahmen, wie zum Beispiel im KIAM geschehen. 
Tabelle 1: Zuordnung der Akzeptanzmodelle zu den Schritten des erweiterten Diffusionsprozesses

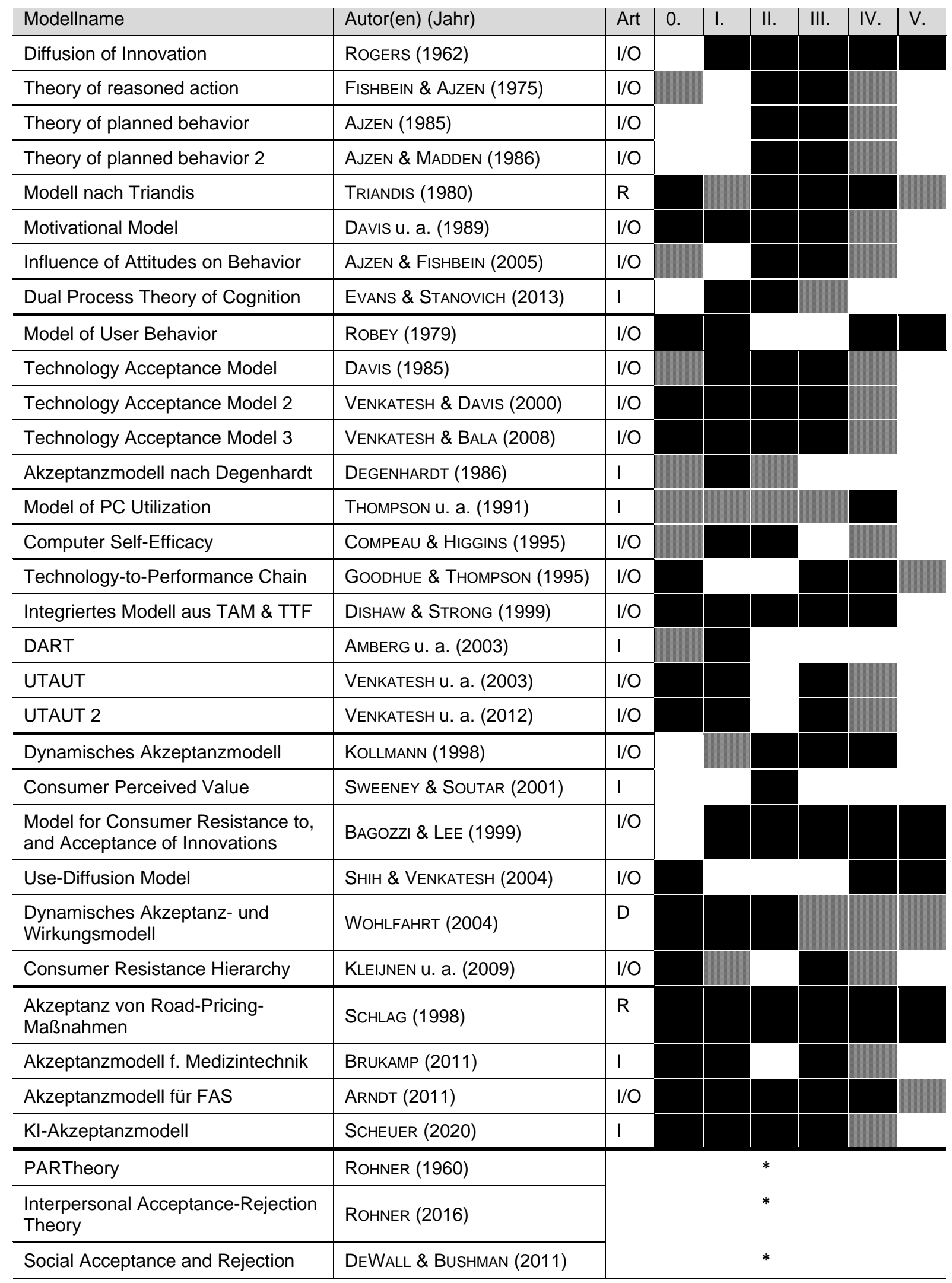

* Zuordnung aufgrund der Sonderstellung nicht sinnvoll

Schritt wird umfänglich erklärt

Schritt wird teilweise erklärt 


\section{Erklärung der Akzeptanzbildung in der Produktentwicklung}

Grundlage für die Erklärung von Akzeptanzbildung in der Methodenanwendung bildet das Diffusionsmodell nach ROGERS [7], [13], da dieses nicht nur den Prozess der Akzeptanzbildung beschreibt, sondern auch erlaubt, den Betrachtungskontext zu konkretisieren. Rogers versteht den Diffusionsprozess dabei als einen Kommunikationsprozess. Neuartige Lösungen werden in einem sozialen System, hier der Entwicklungsorganisation, über verschiedene Kanäle an die Mitglieder der Entwicklungsorganisation kommuniziert. Unter dieser Annahme lassen sich die Beziehungen zwischen Akzeptanzobjekt, -subjekt und -kontext, welches auf ein Modell nach LUCKE [42] zurückgeht, wie in Abbildung 1 dargestellt, konkretisieren.

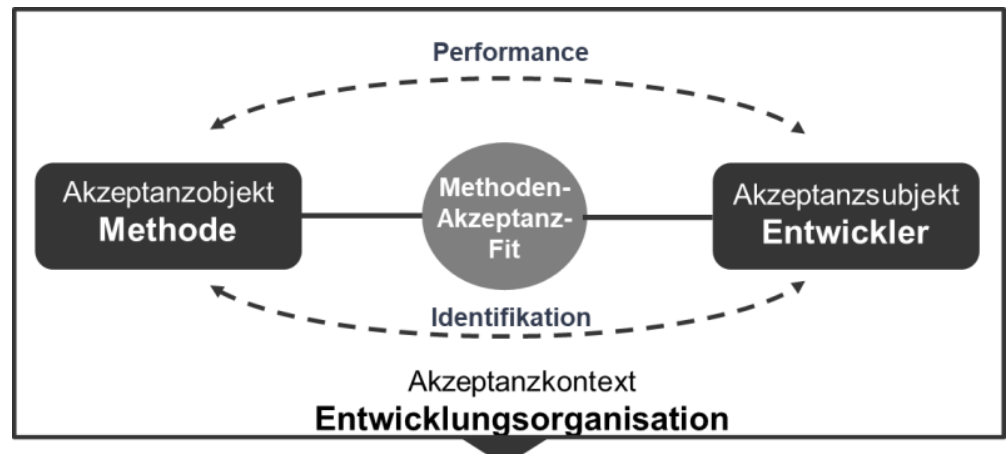

Akzeptanz

Abbildung 1: Beziehungen zwischen Akzeptanzobjekt, -subjekt und -kontext in Anlehnung an LUCKE [42]

Akzeptanzobjekt sind Methoden, die im Rahmen der Produktentwicklung Verwendung finden und im Sinne einer technischen Assistenz den Entwickler in konkreten Arbeitssituationen unterstützen sollen. Methoden sind für die Entwicklung deswegen von Bedeutung, weil sie wesentlich zur Effektivität und Effizienz in der Entwicklung beitragen. Der Grund für die Entwicklung von Methoden liegt im Allgemeinen darin, dass Probleme erkannt werden, die mit bekannten Lösungsmechanismen nicht mehr zufriedenstellend gelöst werden können. Unternehmen haben aber auch ein Interesse am Einsatz von Methoden, wenn diese im eigenen Entwicklungskontext entstanden bzw. an diesen adaptiert wurden, weil sie maßgeblich dazu beitragen, Entwicklungswissen der Organisation zu verstetigen.

Methoden sind als Handlungsvorschriften präskriptiv. Sie beinhalten formalisierte Beschreibungen für bestimmte Aktivitäten mit operativem Charakter, die klären, wie etwas zu tun ist und geben damit dem Vorgehen bei diesen Aktivitäten einen Handlungsrahmen [43]. Methoden unterstützen dabei entweder Aktivitäten des technisch-physikalischen Entwickelns (Analyse, Synthese, Bewerten) oder des technischen Managements, indem Daten und Informationen gezielt für Entwicklungsaufgaben und Entscheidungen bereitgestellt werden. Während Aktivitäten im Rahmen der Synthese beziehungsweise Analyse als Kernaufgaben von Ingenieuren betrachtet werden können, hat die Verwaltung, Beschaffung und Speicherung von Daten und Informationen einen eher administrativen Charakter, der zwar notwendig ist, aber zusätzliche Ressourcen beim Entwickler bindet, die dann nicht mehr für die eigentliche Entwicklungstätigkeit zur Verfügung stehen.

Das Akzeptanzsubjekt ist in diesem spezifischen Fall der Entwicklungsingenieur, der einerseits durch seine Fähigkeiten, Kompetenzen und Erfahrungen charakterisiert wird und andererseits mit den Methoden in definierten Aufgaben unterstützt werden soll. Bei der Auswertung der Akzeptanzmodelle wurde deutlich, dass die Akzeptanz nicht nur von individuellen Faktoren abhängt, sondern auch durch die Art der zu erfüllenden Aufgabe geprägt ist. GODHUE \& THOMPSON beschreiben dieses Phänomen in Ihrer Technology-toPerformance Chain [28] durch den sogennannten Task-Technology Fit generisch als den Grad der Anwendbarkeit der Technologie für das jeweilige Aufgabenset. Bezogen auf die 
Methodenverwendung gilt es also zu hinterfragen, wie und in welcher Art und Weise betrachtete Methoden spezifizierte Entwicklungsaktivitäten unterstützen. Im Rahmen eines Methoden-Aufgaben-Fits (MAF) sind also Anforderungen der Aktivitäten, individuelle Fähigkeiten und die Funktionalität der Methode hinsichtlich ihrer Übereinstimmung zu bewerten [28]. Bezüglich der Funktionalität der Methode gilt es zudem zu beachten, dass diese in der Regel mittels Tools zur Verfügung gestellt werden. Um die genannte Übereinstimmung messen zu können, werden Ansätze benötigt, um die Eignung bzw. Nutzung des Tools von der dahinterliegenden Methode zu trennen.

Zudem wurden in der entwicklungsmethodischen Forschung eine Reihe von Untersuchungen angestellt, um das Problemlösen in der Produktentwicklung aus der Sichtweise des Entwicklers unter Nutzung denkpsychologischer Ansätze zu beschreiben und zu analysieren (z.B. in [44]). BIRKHOFER u. a. [43] erarbeiteten hieraus nicht nur Kompetenzen und Fähigkeiten, die vom Entwickler erwartet werden können, sondern erklären auch das Problemlöseverhalten bzw. die Effekte, wie durch Methodenanwendung kognitive Limitierungen überwunden werden können. Die Erkenntnisse aus diesen Studien gilt es nun mit den die Akzeptanzbildung beschreibenden Einflussfaktoren zusammenzubringen.

Zu guter Letzt spielt der Akzeptanzkontext eine erhebliche Rolle, um Diffusionsprozesse von Methoden als Lösungsansätze für Herausforderungen in der Entwicklung zu verstehen. Den engeren Kontext stellt die vom Entwickler als Individuum zu lösende Entwicklungsaufgabe dar. Den weiteren Kontext bildet die Entwicklungsorganisation als ein soziales System von Individuen, die gemeinsam arbeiten, um unter Nutzung von Hierarchien und Arbeitsteilung ein gemeinsames Ziel zu erreichen [7]. Damit können auch Wechselwirkungen zwischen dem sozialen System und dem Individuum adressiert werden, die den Diffusionsprozess prägen. Die Entwicklungsorganisation soll dabei als ein System aus Entscheidungen verstanden werden, den Ordnungsrahmen bildet Kommunikation, die individuelle Nutzungsentscheidungen und deren organisationale Reaktion darauf strukturiert.

Die Akzeptanzforschung zeigt also sowohl strukturell inhaltliche als auch zeitlich logische Modelle auf, mit deren Hilfe sich die Methodeneinführung samt entsprechender Randbedingungen erklären lässt. Im nächsten Schritt müssen diese theoretischen Erkenntnisse insbesondere unter den Gesichtspunkten der Entwicklungspraxis durch empirische Erhebungen validiert werden.

\section{Zusammenfassung und Ausblick}

Im Ergebnis der vorstehenden Analyse entstand ein Überblick, welche bestehenden Konzepte ohne weiteres auf die Produktentwicklung übertragen werden können und an welchen Stellen zusätzliche Forschungsarbeit geleistet werden muss, um die Steigerung von Methodenakzeptanz in der Praxis zu unterstützen. Hieraus konnte ein erstes Konzept zur detaillierten Untersuchung von Methodenakzeptanz in der Produktentwicklung abgeleitet werden: Eine Nutzungsabsicht manifestiert sich am ehesten dann, wenn der Nutzungsvorteil klar ersichtlich ist. In der Produktentwicklung wird der Erfolg des Methodeneinsatzes bisher allerdings kaum hinreichend und schon gar nicht für alle Stakeholder gleichermaßen evaluiert. Zunächst ist es daher erforderlich, den Status Quo zu erfassen. Hierzu können nun in der Kopplung der prozessorientierten Identifikation von Präskriptoren und der problembezogenen Beschreibung von Akzeptanzobjekt, -subjekt und -kontext die Einflussgrößen für die Beschreibung von Methodenakzeptanz konkretisiert werden. Der Methoden-Aufgaben-Fit bildet in diesem Spannungsfeld damit letztlich eine Brücke zwischen Akzeptanzobjekten und -subjekt. Gleichzeitig determiniert und konkretisiert er einerseits das Nutzererleben und andererseits die Performance der Methodenanwendung. Die damit verbundenen Erklärungsansätze für die Akzeptanz gilt es aus entwicklungsmethodischer Sicht zu interpretieren und zu bewerten, um letztlich die Passung zwischen Methode und Aufgabe konkretisieren zu können. 
Auch müssen die gezeigten Zusammenhänge in der Praxis noch validiert werden, um sogenannte blinde Flecken zu eliminieren. Hierzu gehören vor allem mögliche Verkürzungen in der Beschreibung von Ursache-Wirkungs-Beziehungen, die sich aus falschen oder unvollständigen Suchkriterien ableiten. Es stellt sich zudem die Frage, inwiefern das Spektrum zwischen Nutzung und Nicht-Nutzung von Methoden differenziert werden muss, da im Unternehmenskontext durchaus von einem gewissen Nutzungszwang ausgegangen werden kann. Die dargestellten theoretischen Konstrukte gilt es daher in den nächsten Schritten für verschiedene Kontexte so zu präzisieren, dass sie über empirische Untersuchungen nachgeweisen werden können.

\section{Danksagung}

Die vorgestellte Forschungsarbeit ist Teil des bayerischen Forschungsverbundes „FORCuDE@BEV - Customized Digital Engineering für Bayerische KMU“ und gefördert durch die Bayerische Forschungsstiftung (BFS). Für den Inhalt dieser Publikation sind die Autoren verantwortlich. Die Autoren danken der Bayerischen Forschungsstiftung (BFS) für die finanzielle Unterstützung.

\section{Literaturverzeichnis}

[1] Birkhofer, Herbert ; Kloberdanz, Hermann ; Sauer, Timothy D. ; Berger, Brenda: Why Methods Don't Work and How to Get them to Work. In: Proceedings of EDIProD 2002. Zielona Góra, Poland, 2002, S. 29-36

[2] GausemeIER, Jürgen: Kurzbericht über die Untersuchung Neue Wege zur Produktentwicklung: eine Untersuchung im Rahmenkonzept „Produktion 2000“. 2. Aufl. Paderborn : Heinz-Nixdorf-Institut, Univ.-GH Paderborn, 1998

[3] HILBIG, WINFRIED: Akzeptanzforschung neuer Bürotechnologien. Ergebnisse einer empirischen Fallstudie. In: Office Management Bd. 32 (1984), Nr. 4, S. 320-323

[4] Fishbein, MARTin ; AJzen, ICEK: Belief, Attitude, Intention, and Behavior: An Introduction to Theory and Research, Addison-Wesley series in social psychology. Reading, Mass: Addison-Wesley Pub. Co, 1975 — ISBN 978-0-201-02089-2

[5] Venkatesh, Viswanath ; Davis, Fred D.: A theoretical extension of the technology acceptance model: Four longitudinal field studies. In: Management Science Bd. 46 (2000), Nr. 2, S. 186-204

[6] Evans, Jonathan St. B. T. ; Stanovich, Keith E.: Dual-Process Theories of Higher Cognition: Advancing the Debate. In: Perspectives on Psychological Science : a Journal of the Association for Psychological Science Bd. 8 (2013), Nr. 3, S. 223-241

[7] Rogers, Everett M.: Diffusion of Innovations. 5th ed. New York : Free Press, 2003

[8] FILIPP, HeLmUt: Akzeptanz von Netzdiensten und Netzanwendungen: Entwicklung eines Instruments zur permanenten Akzeptanzkontrolle, Uni Karlsruhe, PhD Thesis, 1996

[9] Kollmann, ToBias: Akzeptanz innovativer Nutzungsgüter und -systeme: Konsequenzen für die Einführung von Telekommunikations- und Multimediasystemen, Neue betriebswirtschaftliche Forschung. Wiesbaden : Gabler, 1998 - ISBN 978-3-409-12849-0

[10] WohlfahrT, Jens: Akzeptanz und Wirkungen von Mobile-Business-Anwendungen, Schriftenreihe Merkur. Hamburg : Dr. Kovač, 2004 — ISBN 978-3-8300-1519-2

[11] DilLeR, H. (Hrsg.): Vahlens grosses Marketinglexikon. 2., völlig überarbeitete und erw. Aufl. München : Beck: Vahlen, 2001 - ISBN 978-3-8006-2689-2

[12] Schäfer, Martina ; KeppleR, DOROTHEe: Modelle der technikorientierten Akzeptanzforschung. In: ZTG Discussion Papers (2013), Nr. 34, S. 87

[13] Rogers, Everett M.: Diffusion of Innovations. New York : Free Press, 1962

[14] RohneR, Ronald P.: Child acceptance-rejection and modal personality in three Pacific societies. Stanford, Stanford University, Unveröffentlichte Thesis, 1960

[15] ROHNER, RONALD P.: Introduction to Interpersonal Acceptance-Rejection Theory (IPARTheory) and Evidence. In: Online Readings in Psychology and Culture Bd. 6 (2016), Nr. 1

[16] AJZEN, ICEK: From Intentions to Actions: A Theory of Planned Behavior. In: KUHL, J. ; BECKMANN, J. (Hrsg.): Action Control: From Cognition to Behavior. Berlin, Heidelberg : Springer Berlin Heidelberg, 1985 — ISBN 9783-642-69746-3, S. 11-39

[17] AJZEn, ICEK ; MAdDEN, ThOMAS J.: Prediction of goal-directed behavior: Attitudes, intentions, and perceived behavioral control. In: Journal of Experimental Social Psychology Bd. 22 (1986), Nr. 5, S. 453-474 
[18] TRIANDIS, H.C.: Values, attitudes, and interpersonal behavior. In: PAGE, M. M. ; SHERIF, C. W. (Hrsg.) Nebraska Symposium on Motivation 1979: Beliefs, attitudes, and values Bd. 27 (1980), S. 195-259

[19] Davis, Fred D. ; Bagozzi, Richard P. ; Warshaw, Paul R.: User Acceptance of Computer Technology: A Comparison of Two Theoretical Models. In: Management Science Bd. 35 (1989), Nr. 8, S. 982-1003

[20] AJZEn, ICEK ; Fishbein, MARTIN: The Influence of Attitudes on Behavior. In: The Handbook of Attitudes. Mahwah, NJ, US : Lawrence Erlbaum Associates Publishers, 2005 — ISBN 978-0-8058-4492-4, S. 173-221

[21] ROBEY, DANIEL: User Attitudes and Management Information System Use. In: Academy of Management Journal Bd. 22 (1979), Nr. 3, S. 527-538

[22] DAVIS, FRED D: A technology acceptance model for empirically testing new end-user information systems: Theory and results, Massachusetts Institute of Technology, PhD Thesis, 1985

[23] Venkatesh, Viswanath ; Bala, Hillol: Technology Acceptance Model 3 and a Research Agenda on Interventions. In: Decision Sciences Bd. 39 (2008), Nr. 2, S. 273-315

[24] Degenhardt, Werner: Akzeptanzforschung zu Bildschirmtext: Methoden u. Ergebnisse : Fischer, 1986

[25] Thompson, Ronald L. ; Higgins, Christopher A. ; Howell, JANE M.: Personal Computing: Toward a Conceptual Model of Utilization. In: MIS Quarterly Bd. 15 (1991), Nr. 1, S. 125

[26] Compeau, Deborah R. ; Higgins, Christopher A.: Application of Social Cognitive Theory to Training for Computer Skills. In: Information Systems Research Bd. 6 (1995), Nr. 2, S. 118-143

[27] Compeau, Deborah R. ; Higgins, Christopher A.: Computer Self-Efficacy: Development of a Measure and Initial Test. In: MIS Quarterly Bd. 19 (1995), Nr. 2, S. 189-211

[28] Goodhue, Dale L. ; Thompson, Ronald L.: Task-Technology Fit and Individual Performance. In: MIS Quarterly Bd. 19 (1995), Nr. 2, S. 213

[29] Dishaw, Mark T ; Strong, Diane M: Extending the technology acceptance model with task-technology fit constructs. In: Information \& Management Bd. 36 (1999), Nr. 1, S. 9-21

[30] AmberG, Michael ; HiRSCHMEIER, MARKUS ; SchOBERT, Deniz: "DART - Ein Ansatz zur Analyse und Evaluierung derBenutzerakzeptan. In: UHR, W. ; EssweIN, W. ; ScHOOP, E. (Hrsg.): Wirtschaftsinformatik 2003: Medien Märkte - Mobilität. Heidelberg : Physica-Verlag, 2003 — ISBN 978-3-7908-0111-8

[31] VeNKATESH ; MoRRIS ; DAVIS ; DAVIS: User Acceptance of Information Technology: Toward a Unified View. In: MIS Quarterly Bd. 27 (2003), Nr. 3, S. 425-478

[32] VenKatesh, VISWANATH ; ThONG, JAmes YL; XU, XIN: Consumer acceptance and use of information technology: extending the unified theory of acceptance and use of technology. In: MIS quarterly, JSTOR (2012), S. 157178

[33] BagozZI, Richard P. ; LeE, KyU-Hyun: Consumer Resistance To, and Acceptance Of, Innovations. In: ARNOULD, E. J. ; ScotT, L. M. (Hrsg.) Advances in Consumer Research Bd. 26 (1999), S. 218-225

[34] SweEney, Jillian C ; Soutar, Geoffrey N: Consumer perceived value: The development of a multiple item scale. In: Journal of Retailing Bd. 77 (2001), Nr. 2, S. 203-220

[35] Shih, Eric Chuan-Fong ; VenKatesh, Alladi: Beyond Adoption: Development and Application of a UseDiffusion Model. In: Journal of Marketing Bd. 68 (2004), Nr. 1, S. 59-72

[36] Kleijnen, Mirella ; Lee, Nick ; WetZels, Martin: An exploration of consumer resistance to innovation and its antecedents. In: Journal of Economic Psychology Bd. 30 (2009), Nr. 3, S. 344-357

[37] SCHLAG, BERNHARD: Zur Akzeptanz von Straßenbenutzungsentgelten. In: Internationales Verkehrswesen Bd. 50 (1998), Nr. 7/8, S. 308-312

[38] DeWall, C. Nathan ; Bushman, Brad J.: Social Acceptance and Rejection: The Sweet and the Bitter. In: Current Directions in Psychological Science Bd. 20 (2011), Nr. 4, S. 256-260

[39] BRUKAMP, KIRSTEN: Akzeptanzfaktoren für die ambulante Telemedizin. In: BRUKAMP, K. ; LARYIONAVA, K. ; SCHWEIKARDT, C. ; Gross, D. (Hrsg.): Technisierte Medizin - Dehumanisierte Medizin? Ethische, rechtliche und soziale Aspekte neuer Medizintechnologien. Bd. 1. Kassel : Kassel University Press, 2011 — ISBN 978-386219-162-8, S. 71-76

[40] ARndt, StePhANiE: Evaluierung der Akzeptanz von Fahrerassistenzsystemen: Modell zum Kaufverhalten von Endkunden: Zugl.: Dresden, Techn. Univ., Diss., 2010, Verkehrspsychologie. 1. Aufl. Wiesbaden : VS Verl. für Sozialwiss, 2011

[41] SChEUER, DenNIS: Entwicklung eines Theoriemodells zur Akzeptanz von Künstlicher Intelligenz. In: Akzeptanz von Künstlicher Intelligenz: Grundlagen intelligenter KI-Assistenten und deren vertrauensvolle Nutzung. Wiesbaden : Springer Fachmedien Wiesbaden, 2020 — ISBN 978-3-658-29526-4, S. 57-65

[42] LUCKE, DORIS: Akzeptanz: Legitimität in der „Abstimmungsgesellschaft“ : Springer, 1995

[43] Birkhofer, H. ; Jansch, J. ; Kloberdanz, H.: An Extensive and Detailed View of the Application of Design Methods and Methodology in Industry. In: SAmUEL, A. E. ; LeWIS, W. (Hrsg.): Proceedings of the International Conference on Engineering Design, 2005, S. 518-523

[44] RuTZ, A.: Konstruieren als gedanklicher Prozess. München, TU München, Dissertation, 1985 